\title{
On Some Applications of Nonlinear Differential Equations in Image Processing: Concepts and Electronic Implementation
}

\author{
S. Morfu ${ }^{1}$ and P. Marquié ${ }^{2}$ \\ Université de Bourgogne, LE2I - CNRS UMR 5158 \\ Dijon, France
}

\section{Introduction}

Quality control by visual inspection usually involves acquisition of images corrupted by noise and sometimes weakly contrasted. That is why image filtering is the first task commonly performed after data acquisition to take a decision in most quality control applications. Since this crucial filtering step allows to obtain a better classification in pattern recognition, the development of new processing algorithms still remains an opened research direction. In image processing field, unconventional methods of filtering inspired by the properties of nonlinear systems have recently been proposed as a convenient solution to solve problems of high computational complexity Teuscher \& Adamatzky (2005). In fact, all these

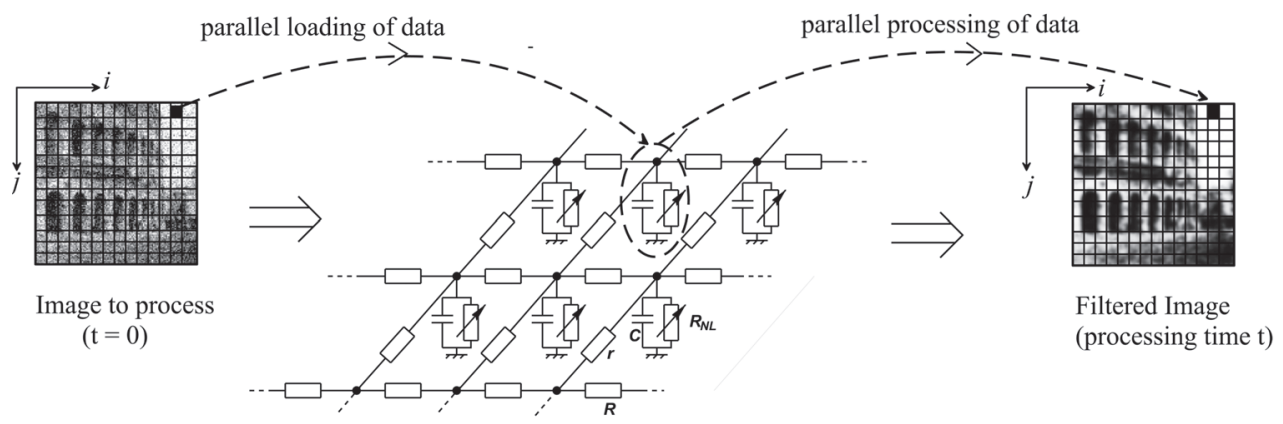

Fig. 1. The concept of image filtering using circuits ruled by nonlinear equations. The voltage at the nodes of the 2 dimensional network obeys to a set of $N \times M$ differential equations where $N \times M$ represents the image size. The initial condition loaded at each node of the network is denoted by the arrow in broken lines and corresponds to the gray level of each pixel.

unconventional algorithms are based on the properties of Nonlinear Differential Equations (NDEs) which can allow to explain various natural phenomena, such as the recurrence of ice ages, the propagation of blood pressure in arteries or the transport mechanisms in living cells to cite but a few. Another interesting feature of these NDEs is that they can also describe the voltage evolution at the nodes of a 2 dimensional electronic network. Therefore, the processing tools derived from the properties of these NDEs can potentially be implemented directly after 
image acquisition. This fact represents a genuine advantage to develop intelligent embedded systems combining acquisition and processing.

Even if such embedded systems are actually far from being operational, this paradigm of nonlinear circuits ruled by NDEs has been raised since the eighties by L.O. Chua and L. Yang under the well known acronym " $C N N$ " which means Cellular Neural (or Nonlinear) Network Chua (1999).

The principle of image filtering with these electronic networks is summarized in fig. 1:

- Each pixel of the initial image is considered as a cell (namely an electronic circuit) coupled to its neighbors. The processing task is set by the properties of this cell, that is by the nonlinear differential equations which rule the 2 dimensional network.

- The initial condition applied to each cell corresponds to the initial gray level of the image to process. Therefore, each cell can be viewed as a processor working in parallel.

- The dynamic of the nonlinear network is interpreted as a specific filtering process. Indeed, the image for a processing time $\breve{a}^{\prime \prime} t$ "ă is obtained noting the state of the whole network at that time " $t$ ". Therefore, the implemented filter is recursive since the filtered image for a given processing time is deduced from the temporal evolution of the network.

This definition reveals many important features of the CNNs such as their parallel architecture, which is suitable for image processing, their strong dependence on initial conditions, offering a rich variety of potential processing and their intrinsic speed which allows real time applications Chua (1999); Chua \& Yang (1988). Since their introduction as a novel class of information processing systems Chua (1999), the CNNs have been the subject of a growing interest with various applications in quality control Morfu et al. (2007); Occhipinti et al. (2001), image compression Venetianer et al. (1995) or image processing Julian et al. (2002); Morfu (2005). To summarize these two decades devoted to CNNs, finding new processing algorithms Grassi et al. (2006); Grassi \& Grieco (2003) and designing the elementary cells of these electronic nonlinear networks Arena et al. (2003); Nagy \& Szolgay (2003) constitute the two main directions of research to develop promising applications using CNNs.

In this chapter, the concept of processing with these CNNs ruled by NDEs will be intensively discussed. Especially, we will restrict this chapter to the study of the properties of NDEs for image processing purposes. More precisely, among the standard processing tools which can be performed with the properties of these NDEs, we propose to detail contrast enhancement, noise filtering or image segmentation, which constitute the most common pre-processing tasks performed to enhance pattern recognition and classification applications. We close this chapter by a slight discussion on a possible implementation of these NDEs.

\section{Reaction-Diffusion electrical networks}

\subsection{Generic presentation}

These kind of networks can be implemented following the electrical structure depicted in figure 2 .

The voltage $U_{i, j}$ at cell $(i, j)$ of the network obeys the standard Nagumo equation:

$$
C \frac{d U_{i, j}}{d \tau}=\frac{1}{R}\left[\sum_{(k, l) \in N_{r}}\left(U_{k, l}-U_{i, j}\right)\right]-I_{N L}\left(U_{i, j}\right)
$$

where $\tau$ corresponds to time and $I_{N L}\left(U_{i, j}\right)$ represents a voltage-dependant nonlinear current. Finally, $\mathrm{Nr}$ denotes the set of nearest neighbors of cell $(i, j)$. 
In the following, we consider two different types of nonlinear current obtained while considering two different nonlinear resistances.

1. The first nonlinear resistor model presents a cubic nonlinear current-voltage characteristics since the nonlinear current $I_{N L}$ obeys

$$
I_{N L}\left(U_{i, j}\right)=U_{i, j}\left(U_{i, j}-a\right)\left(U_{i, j}-b\right) /\left(R_{0} a b \beta\right)
$$

In this case, setting:

$$
\alpha=\frac{b}{a}, \quad X_{i, j}=\frac{U_{i, j}}{b}, \quad D=\frac{R_{0} \alpha \beta}{R}, \quad t=\frac{\tau}{R_{0} \alpha C \beta},
$$

leads to the well known normalized Nagumo equation:

$$
\frac{d X_{i, j}}{d t}=D\left[\sum_{(k, l) \in N_{r}}\left(X_{k, l}-X_{i, j}\right)\right]+f\left(X_{i, j}\right), \quad \text { with } \quad f\left(X_{i, j}\right)=-X_{i, j}\left(X_{i, j}-\alpha\right)\left(X_{i, j}-1(\not 4)\right.
$$

2. The second nonlinear resistor model presents a current-voltage characteristics approximating a sine shape for voltages belonging to the interval $[-2 \mathrm{~V}, 2 \mathrm{~V}]$, that is

$$
I_{N L}\left(U_{i, j}\right)=\frac{16 \psi}{R_{0}} \sin \left(2 \pi U_{i, j}\right) \simeq P(U) / R 0 .
$$

In this expression, after the use of a least-square method, $\mathrm{P}(\mathrm{U})$ is a fifteenth order polynomial approximating the sine law (5) on the $[-2 \mathrm{~V} ; 2 \mathrm{~V}]$ voltage range. As for the first type of nonlinear resistor, a feedback resistor $R_{0}$ between the input and the output of the nonlinear source allows to obtain the desired nonlinear current by applying the Ohm law to this resistor $R_{0}$.

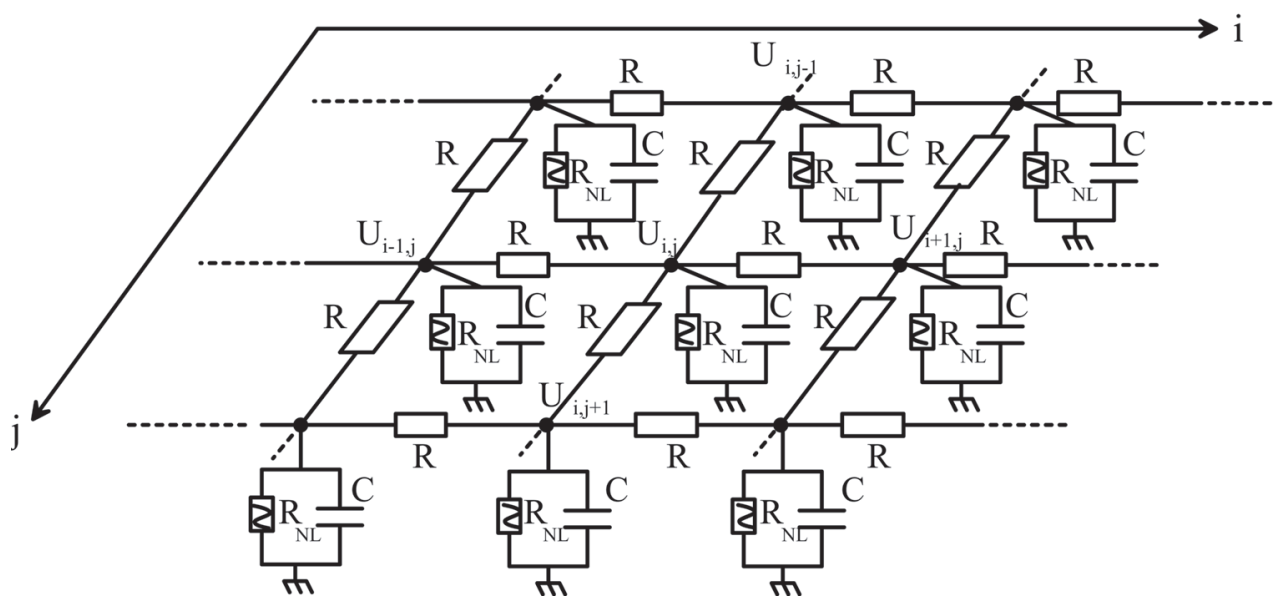

Fig. 2. The Reaction-Diffusion electrical network. $R$ and $C$ respectively represents a linear resistor and capacitor while $R_{N L}$ is the nonlinear resistor whose current voltage caracteristic obeys to one of the two laws (2) or (5). 


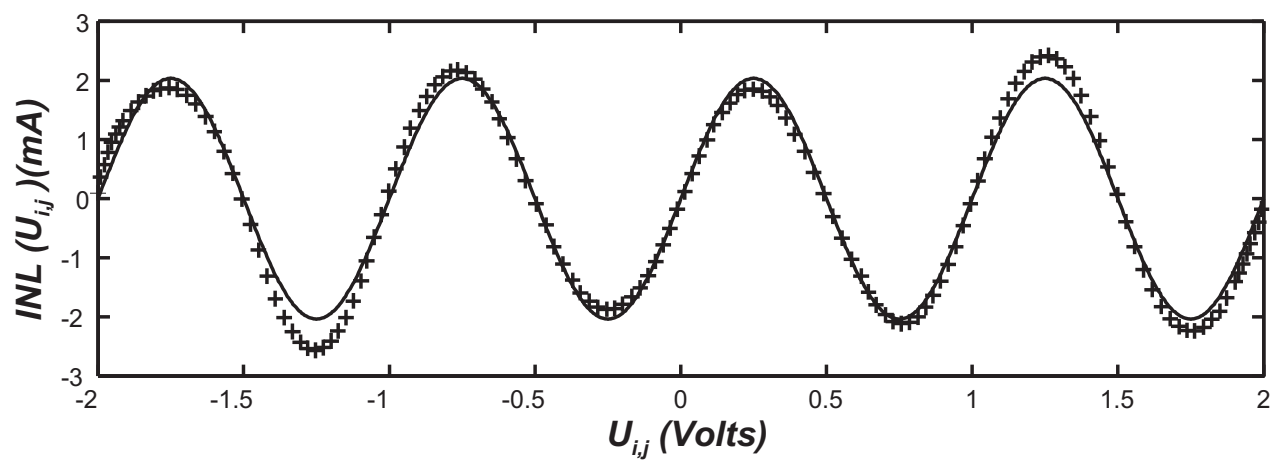

Fig. 3. Nonlinear sinusoidal current-voltage characteristics. The experimental current $(+$ signs) perfectly matches the theoretical law (5) on the $[-2 \mathrm{~V}, 2 \mathrm{~V}]$ voltage range. $R_{0}=2 \mathrm{~K} \Omega$, $\psi=0.25 \mathrm{~V}$.

As shown in figure 3, the experimental nonlinear current-voltage characterisics (cross signs) perfectly fits the theoretical sine law given by (5) (solid line.)

Next, setting the following transformations

$$
\tau=t R_{0} C, \quad D=\frac{R}{R_{0}}, \quad U_{i, j}=4 X_{i, j}-2,
$$

we get the normalized reaction diffusion equation

$$
\frac{d X_{i, j}}{d t}=D\left[\sum_{(k, l) \in N_{r}}\left(X_{k, l}-X_{i, j}\right)\right]+g\left(X_{i, j}\right) \quad \text { with } \quad g\left(X_{i, j}\right)=-4 \psi \sin \left(8 \pi X_{i, j}\right)
$$

\subsection{Image processing applications}

\subsubsection{Image segmentation}

For image processing application purpose, the properties of both (4) and (7) systems have been investigated. Their behavior can be understood while using the following mechanical analogy. Indeed, from a mechanical point of view, each of these two systems corresponds to a two-dimensional network of $N \times M$ particles of unit mass in the overdamped regime, coupled together with springs of strengh $D$. Each particle is submitted to a nonlinear force, $f$ or $g$ deriving from a potential $\Phi(x)=-\int_{0}^{x} f(x) d x$ or $\Phi(x)=-\int_{0}^{x} g(x) d x$, according to the considered nonlinear resistor model.

This mechanical analogy can be highlighted by applying the fundamental principle of dynamics to the network of particles presented in figure 4 .

Indeed, if $X_{i, j}$ denotes the displacement of the particle $(i, j)$, submitted to the potential $\Phi$ of figure $4 .(b)$, the fundamental principle of dynamics writes

$$
\frac{d^{2} X_{i, j}}{d t^{2}}=-\frac{d X_{i, j}}{d t}+D\left[\sum_{(k, l) \in N_{r}}\left(X_{k, l}-X_{i, j}\right)\right]-\frac{d \Phi}{d X_{i, j}}
$$

where $-\frac{d X_{i, j}}{d t}$ corresponds to the friction force, while $\frac{d^{2} X_{i, j}}{d t^{2}}$ represents the inertia term. Equations (4) and (7) are obtained while neglecting the inertia term versus the friction force, 
(a)

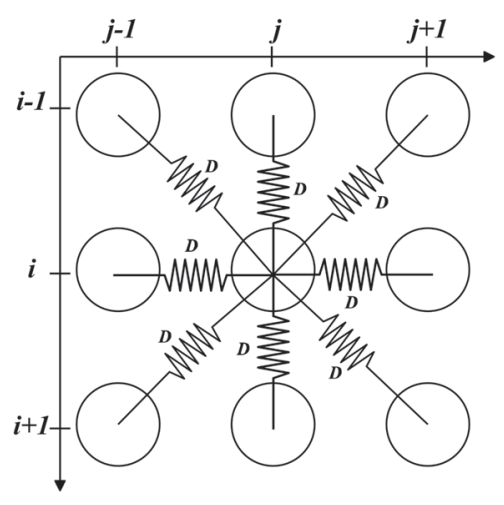

(b)
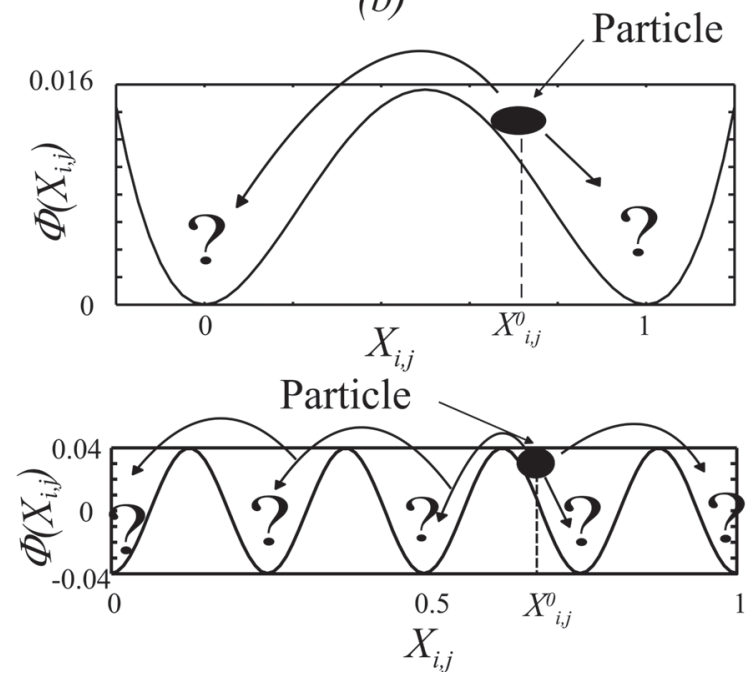

Fig. 4. Mechanical analogy of systems (4) and (7). (a) 2-D network of particles coupled by springs of strengh $D$ and submitted to one of the two potentials represented in $(b)$. Depending on both its initial condition and the resulting springback forces, each particle will be attracted by one of the different potential wells of figure $(b)$.

which corresponds to the overdamped regime. The stability analysis of the two systems (4) and (7) depends respectively on the considered nonlinear forces $f$ and $g$, the potentials they derive from being represented in figure 4. $(b)$. This analysis being presented in details in Morfu et al. (2008), we just present here some remarks allowing to understand the dynamics of our system:

1. Local minima of the potential correspond to the stable states of the system, while the local maxima define the unstable states. Accordingly, in the uncoupled case $(D=0)$, a particle (or state of a cell or grey level) will evolve towards one of the stable states, depending on its initial condition versus the position of the unstable states acting like thresholds.

2. From a mechanical point of view, the term $D\left[\sum_{(k, l) \in N_{r}}\left(X_{k, l}-X_{i, j}\right)\right]$ corresponds to the neighbors-induced resulting elastic force acting on the particle $(i, j)$. In the coupled case, $(D \neq 0)$, this particle will evolve towards one of the stable states, not only depending on its initial condition but also on the resulting elastic force.

From an image processing point of view, it can be concluded that if an image is loaded as initial condition in the electrical network with cubic nonlinearity obeying to (4), the latter will evolve, for sufficiently large time, to an almost black and white picture, since only two stable states exist, corresponding respectively to 0 (black) and 1 (white).

Next, considering a sinusoidal nonlinearity (5), the system may evolve towards a larger number of stable states defined by the shape of the associate potential. It will then be possible to get a segmentation of the initial image into several regions related to the mean grey levels corresponding to the system stable states.

As an example, we present, in the context of quality control, the result of an image segmentation realized with a nonlinear network using respectively cubic and sinusoidal 


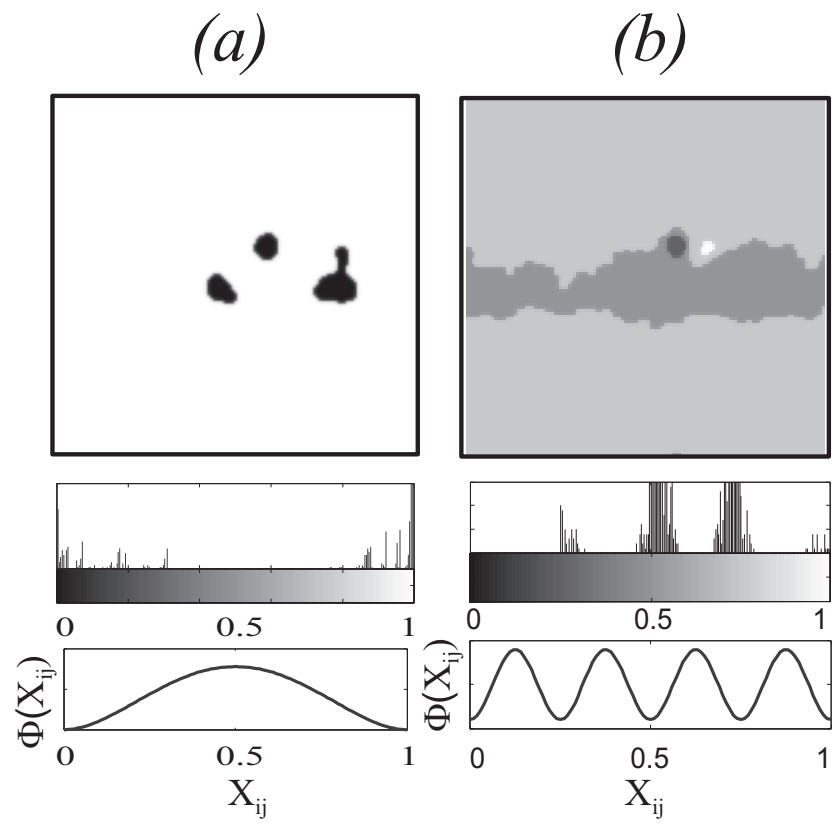

Fig. 5. Image segmentation using electrical reaction-diffusion networks. (a) The use of a cubic nonlinearity does not allow to extract the regions of interest. Indeed, as seen in the histogram and the potential under the image resulting from the segmentation process, the image grey levels are mainly localized in the two potential wells, corresponding respectively to black (0) and white (1). Parameters: $D=0.05, \alpha=0.5, t=5000$. (b) The sinusoidal nonlinearity enables the extraction of the four regions of interest as observed on the image and on the grey levels repartition in four potential wells. Parameters: $D=1.6$ and $t=5000$.

nonlinearity. The initial noisy and weak contrasted image, considered in Morfu et al. (2008), represents a digitalized radiography of a soldering between two rods of metal. This image reveals the following four regions of interest:

1. The background of the image (light grey) which corresponds to the two rods of metal

2. The central part of the image which corresponds to the soldered joint (medium grey)

3. A dark grey spot localized in the soldered joint and corresponding to a gaseous inclusion

4. A projection of metal (white spot)

As seen in figures 5.(a) and $(b)$, if the use of a cubic nonlinearity does not allow to extract the four specific regions, the sinusoidal nonlinearity enables to reveal the four individual components of the original noisy and weak contrasted image.

\subsubsection{Noise filtering and contrast enhancement}

During the last decades, a particular interest has been devoted to the development of bio-inspired processing tasks in the field of signal or image processing as seen in the previous section. In particular, it has been shown that noise filtering operations based on reaction-diffusion systems with cubic nonlinearity could be realized. Nevertheless, due to the 


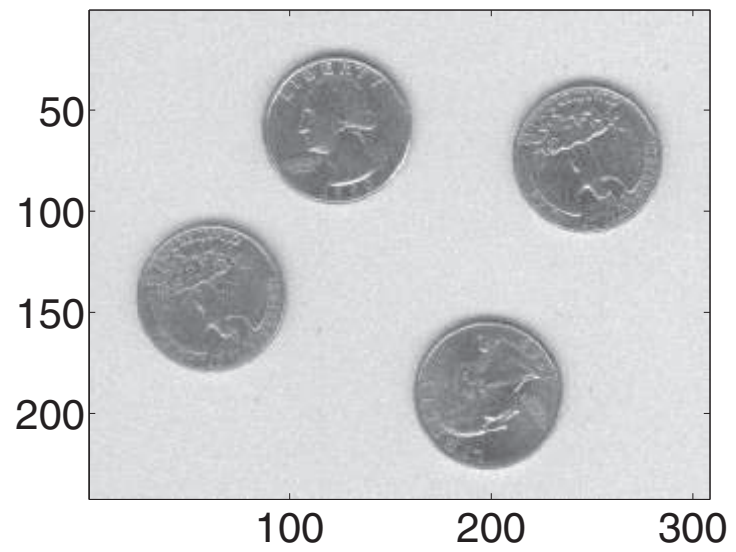

Fig. 6. Initial noisy image

homogeneous or isotropic diffusion process, this noise removal operation provides a blurry filtered image, which renders difficult the precise edge or contour location. As an example, in this section, we will consider the initial noisy image presented in figure 6 .

In order to improve the noise filtering efficiency, the following diffusion process has been proposed Morfu (2009):

$$
\frac{d X_{i, j}}{d t}=\frac{D}{\left|N_{r}\right|}\left[\sum_{(k, l) \in N_{r}} h\left(\left\|X_{k, l}-X_{i, j}\right\|\right) \times\left(X_{k, l}-X_{i, j}\right)\right]-\gamma X_{i, j}\left(X_{i, j}-\alpha\right)\left(X_{i, j}-1\right),
$$

where $|N r|$ represents the total number of neighbors for the considered pixel $(i, j)$.

The respective effects of the cubic nonlinearity on one hand, and of the anisotropy depicted by the function $h$ on the other hand can be considered separately in equation (9).

Indeed, according to the weight $\gamma$ of the cubic nonlinearity with respect to the function $h$, the following diffusion process may be highlighted:

\section{Isotropic and linear diffusion process.}

When the nonlinear parameter $\gamma$ is set to 0 and when the $h$ function takes the value 1 , then equation (9) corresponds to the classical linear heat equation. The resulting filtered image is represented in figure 7. (a) for the processing time $t=6$. Furthermore, the profile of line number 50 is also shown for different processing times. It can be observed that, as time goes on, the noise effect tends to disappear but in the same time, the image details like the contours are also removed, which gives a blurry processed image.

\section{Isotropic and purely nonlinear process}

When the $h$ function takes the value 1, equation (9) reduces to the standard Nagumo equation (4) which describes a nonlinear process. The resulting filtered image and the profiles versus time of the line number 50 are presented in figure 7.(c) et $(d)$. As the processing time increases, a contrast enhancement occurs (the relative amplitude of the profile increases) due to the cubic nonlinearity. However, as the process is isotropic, the contour location is not preserved since the resulting image remains blur.

\section{Purely anisotropic diffusion process}

When $h \neq 1$ and $\gamma=0$ (which means that there is no more nonlinearity in the system), one 
get the Peronna and Malik algorithm allowing to overcome the blur effect due to isotropy Perona \& Malik (1990). The main idea consists in replacing the diffusion coefficient $D$ by a function $h$ of the gradient amplitude, which corresponds to a modulation of the diffusion depending on the different regions of the considered image. More precisely:

- for regions containing edges or contours, diffusion is stopped by setting the $h$ function to zero. Indeed, a contour corresponds to an important value of the local gradient. For this reason, in equation (9), the function $h$ will depend on the local gradient modulus, which can be approximated by $\left\|X_{k, l}-X_{i, j}\right\|$ for the pixel $(i, j)$ with neighor of index $(k, l)$.

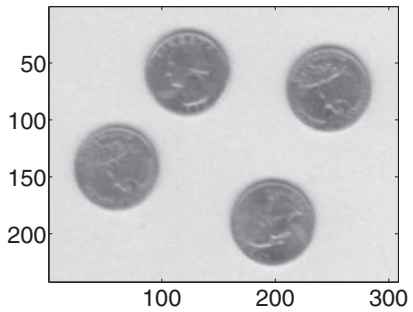

(a)

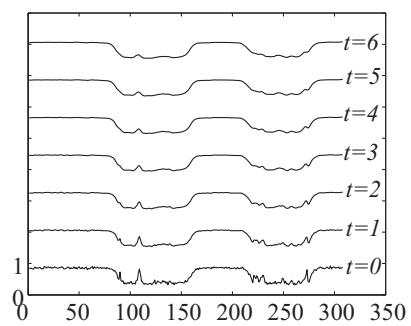

(d)

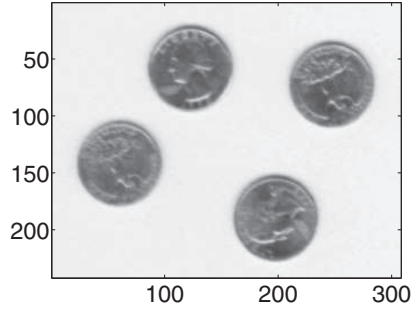

(b)

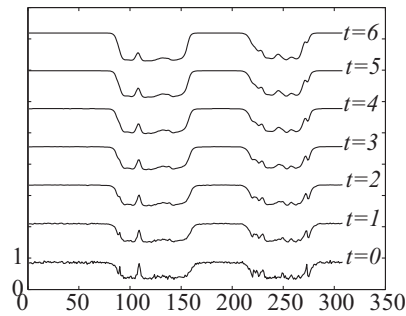

(e)

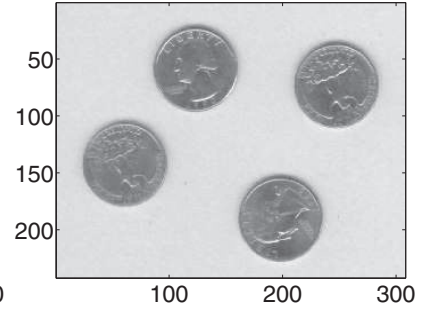

(c)

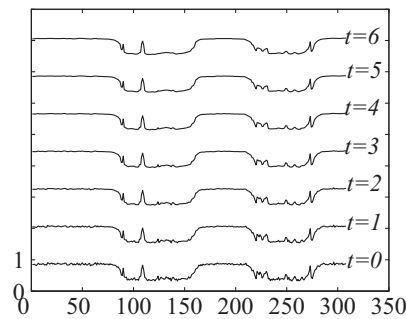

(f)

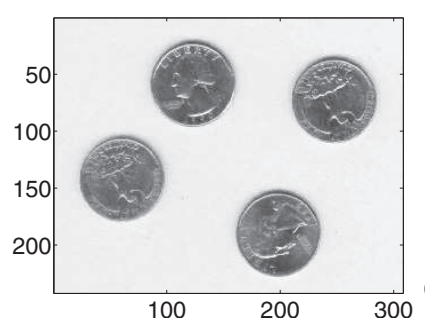

(g)

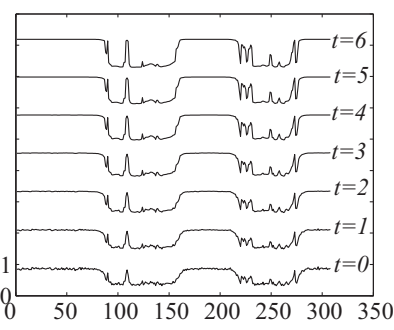

(h)

Fig. 7. : Image processing with different diffusion processes. $(a)$ and $(d)$ Isotropic and linear process $(h=1$ and $\gamma=0)$. (b) and (e) Isotropic and nonlinear process $(h=1, \gamma=1$ and $\alpha=0.5)$. (c) and $(f)$ Purely anisotropic process $(\sigma=0.03783, \gamma=0)$. $(g)$ and $(h)$ Anisotropic and nonlinear process $(\sigma=0.03783, \gamma=1$ and $\alpha=0.5)$. In each case, the filtered image is obtained for the processing time $t=2$ after integrating the system of nonlinear differential equations (9). Profiles of the line number 50 are also represented for different processing times. 
- for homogeneous regions corrupted by noise, as the local gradient is quite weak, the diffusion process can be enhanced for eliminating the noise by the increase of the $h$ function.

From these remarks, it comes that the $h$ function must be a monotonic decreasing function versus the local gradient, from 1 which corresponds to the maximum of diffusion effect to 0 corresponding to no diffusion effect. As an example, Peronna and Malik have considered the following Lorentz function in their studies Perona \& Malik (1990):

$$
h\left(\left\|X_{k, l}-X_{i, j}\right\|\right)=\frac{D}{1+\left(\frac{\left\|X_{k, l}-X_{i, j}\right\|}{\sigma}\right)^{2}},
$$

where the parameter $\sigma$ adjusts the decreasing of the function $h$, and then the weight of anisotropy. So it can be set in order to preserve the edges in the considered image.

According to Black et al. (1998), this parameter represents a noise estimator defined by

$$
\sigma=1.4826 M A D(\nabla X) / \sqrt{2}
$$

where $M A D$ and $\nabla X$ denote the median absolute deviation and the gradient of the image to be processed, respectively.

Considering the example of figure 6, the parameter $\sigma$ has been estimated to $\sigma=0.03783$.

As observed in figures 7.(e) and $(f)$, this diffusion process allows noise removal but without any contrast enhancement.

\section{Process combining anisotropic and nonlinear diffusion}

When $\gamma \neq 0$ and when the function $h$ obeys to (10), the benefits of both anisotropy and nonlinearity are combined, as verified in figures 7. $(g)$ et $(h)$. Indeed, the obtained processing results clearly show that noise is removed while the image contrast is enhanced Morfu (2009).

\section{Hamiltonian electronic processing systems}

\subsection{Theoretical background}

As for the reaction diffusion processing system, we can use a mechanical analogy to depict the electrical network. So we consider a two-dimensional network of $N \times M$ particles of unit mass, but contrary to the previous case, the friction force is neglected versus the inertia term in equation (8). Furthermore, we will consider that all the particles are uncoupled, which corresponds to $D=0$. Under these conditions, the oscillations of the $N \times M$ particles obey the following system of differential equations:

$$
\frac{d^{2} W_{i, j}}{d t^{2}}=f\left(W_{i, j}\right) \quad i=1,2 \ldots N \text { et } j=1,2 \ldots M,
$$

with the the nonlinear force $f$ which can be written under the following form

$$
f\left(W_{i, j}\right)=-\omega_{0}^{2}\left(W_{i, j}-m-\alpha\right)\left(W_{i, j}-m+\alpha\right)\left(W_{i, j}-m\right) .
$$

This force $f$ derives from the potential $\Phi$ represented on figure 8 . The roots $m-\alpha$ and $m+\alpha$ of the cubic force define the position of the two potential wells, while the root $m$ indicates the position of the potential barrier height $\Delta$. From a theoretical point of view, as seen in figure 8 , if the potential energy of a particule (or oscillator) is greater than the potential barrier height, as no friction force is considered, this particle will oscillate without any damping that is for an infinite time. 


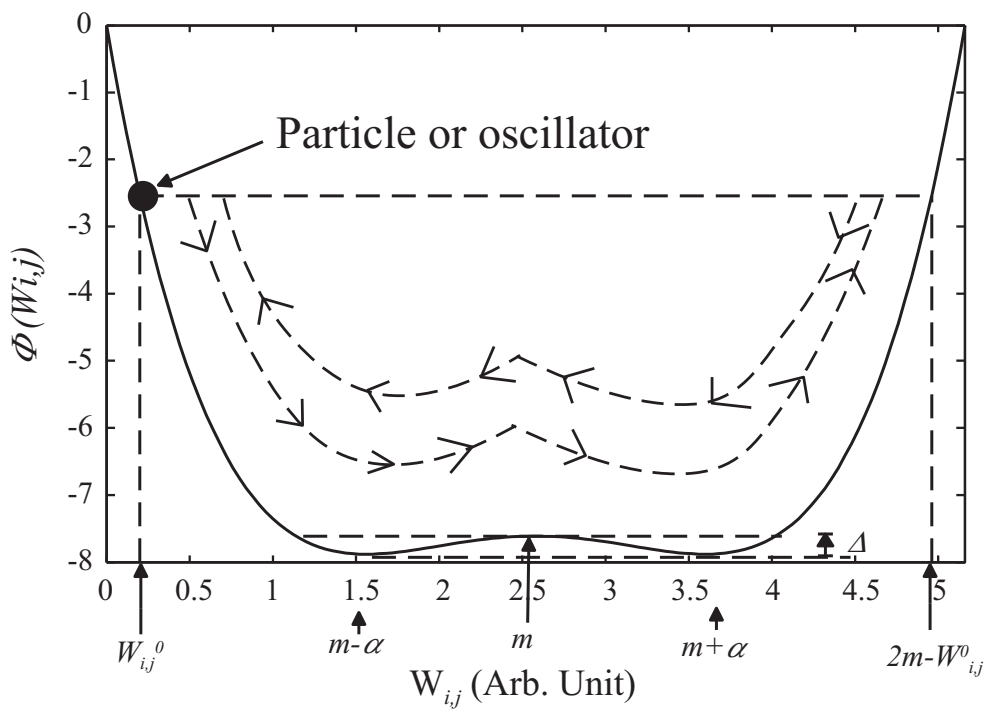

Fig. 8. Potential related to the nonlinear force (13) represented for $m=2.58, \alpha=1.02$ and $\omega_{0}=1$. As indicated by the dot line, a particle (or oscillator) which initial condition $W_{i, j}^{0}$ is greater than $m-\alpha \sqrt{2}$, oscillates in a symetric way, crossing periodically the potential barrier. $\Delta$.

\subsection{Electronic implementation of an oscillator}

The schematic electronic diagram of this kind of elementary nonlinear oscillator is represented in figure 9. It is composed of:

- 3 external sources adjusting the three roots of the cubic nonlinearity $m, m-\alpha$ and $m+\alpha$.

- 2 AD633JNZ analog multipliers with scale factor $1 / 10 \mathrm{~V}^{-1}$.

- 2 inverting amplifiers with gain $-K=-10$ for the compensation of the multipliers scale factors.

- a double integrator with time constant $R C$ assuring a feedback between the input and the output of the nonlinear circuit.

- a $1 N 4148$ diode with threshold voltage $V_{T}$, used to apply the initial condition $W_{i, j}^{0}$ to the considered cell $(i, j)$.

The diode cathode potential $W_{i, j}$ has to be expressed in order to get the nonlinear differential equation (12).

The output of the second amplifier, which can be written under the following polynomial form

$$
P\left(W_{i, j}\right)=\left(W_{i, j}-m-\alpha\right)\left(W_{i, j}-m+\alpha\right)\left(W_{i, j}-m\right),
$$

is connected to the input of the circuit via the double integrator, which leads to the expression of the cathode potential: 


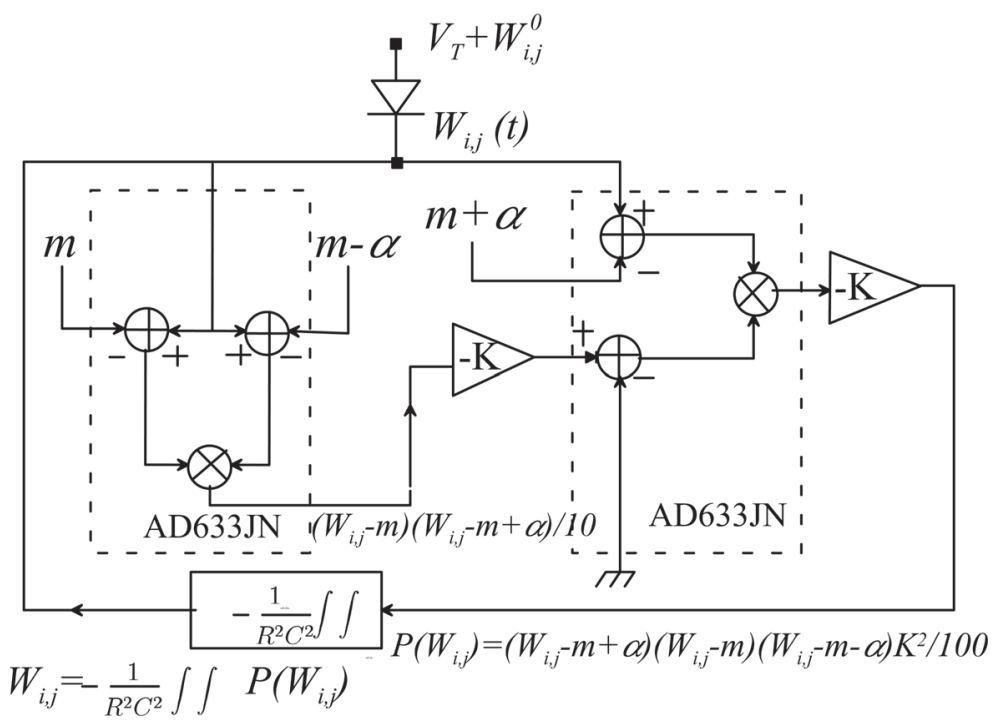

Fig. 9. Sketch of the nonlinear oscillator circuit obeying to (12). The component values are: $R=10 \mathrm{~K} \Omega$ and $C=10 \mathrm{nF} .-\mathrm{K}=-10$ stands for the scale factor of the inverting amplifiers.

$$
\begin{array}{r}
W_{i, j}=-\frac{K^{2}}{100 R^{2} C^{2}} \iint\left(W_{i, j}-m+\alpha\right)\left(W_{i, j}-m-\alpha\right)\left(W_{i, j}-m\right) d t \\
\text { with } i=1,2 \ldots N \text { and } j=1,2 \ldots M .
\end{array}
$$

Deriving twice equation 15 gives the nonlinear differential equation

$$
\frac{d^{2} W_{i, j}}{d t^{2}}=-\frac{K^{2}}{100 R^{2} C^{2}}\left(W_{i, j}-m+\alpha\right)\left(W_{i, j}-m-\alpha\right)\left(W_{i, j}-m\right) .
$$

which exactly corresponds to the normalized equation (12) provided that $\omega_{0}=K /(10 R C)$.

\subsection{Image processing based on the properties of the nonlinear oscillators}

Solutions of equation (16) can be obtained and written under the form:

$$
W_{i, j}(t)=m+\left(W_{i, j}^{0}-m\right) \operatorname{cn}\left(\omega_{i, j} t, k_{i, j}\right),
$$

where $W_{i, j}^{0}$ corresponds to the initial condition applied to the particle $(i, j)$ of the network, which corresponds to the grey level of the pixel $(i, j)$ in the image processing context.

In equation (17), cn corresponds to the Jacobi elliptic function, which modulus $k_{i, j}$ and pulsation $\omega_{i, j}$ are given by

$$
\omega_{i, j}\left(W_{i, j}^{0}\right)=\omega_{0} \sqrt{\left(W_{i, j}^{0}-m\right)^{2}-\alpha^{2}} \quad \text { and } \quad k_{i, j}\left(W_{i, j}^{0}\right)=\frac{1}{2} \frac{\left(W_{i, j}^{0}-m\right)^{2}}{\left(W_{i, j}^{0}-m\right)^{2}-\alpha^{2}} .
$$


Pulsation and shape of oscillations are imposed by the modulus of the Jacobi elliptic function. They depend then on parameters $\alpha$ and $\omega_{0}$ of the nonlinearity, but also on the initial condition $W_{i, j}^{0}$.

Because of the sensitivity to initial condition in this kind of nonlinear system, two oscillators with a very weak difference of initial condition will rapidly present a phase opposition. Indeed, considering respectively the initial conditions 0 and 0.2 for the oscillators of figure 10 , phase opposition is attained theoretically at time $t_{\text {opt }}=1.64 \mathrm{~ms}$ :

For this time $t_{\text {opt }}$, the initial weak difference between the amplitudes of the two oscillators becomes the largest one since it is equal to 5.1 (see figure 11).

The experimental results obtained using the electrical circuit present a good agreement with the theoretical predictions, despite the slight discrepancy for the optimal time $(1.46 \mathrm{~ms}$ instead of $1.64 \mathrm{~ms}$ ), mainly imputable to the components uncertainties. Let us note that the amplitude difference $\delta$ of the oscillators evoves with time, reaching periodically local minima and maxima.

The dynamics of this oscillators network can be understood in term of image processing. Considering indeed a weak contrasted image as initial condition, if the grey scale of the original image belongs to the interval $[0 ; 0.2]$, then the amplitude difference between the two oscillators will follow the theoretical curve presented in figure 11. Consequently, as shown in figure 12, the particular times for which the amplitude difference vanishes will define the minima of contrast. On the contrary, the particular times corresponding to local maxima of $\Delta$ may lead to contrast enhancement with possible image inversion.
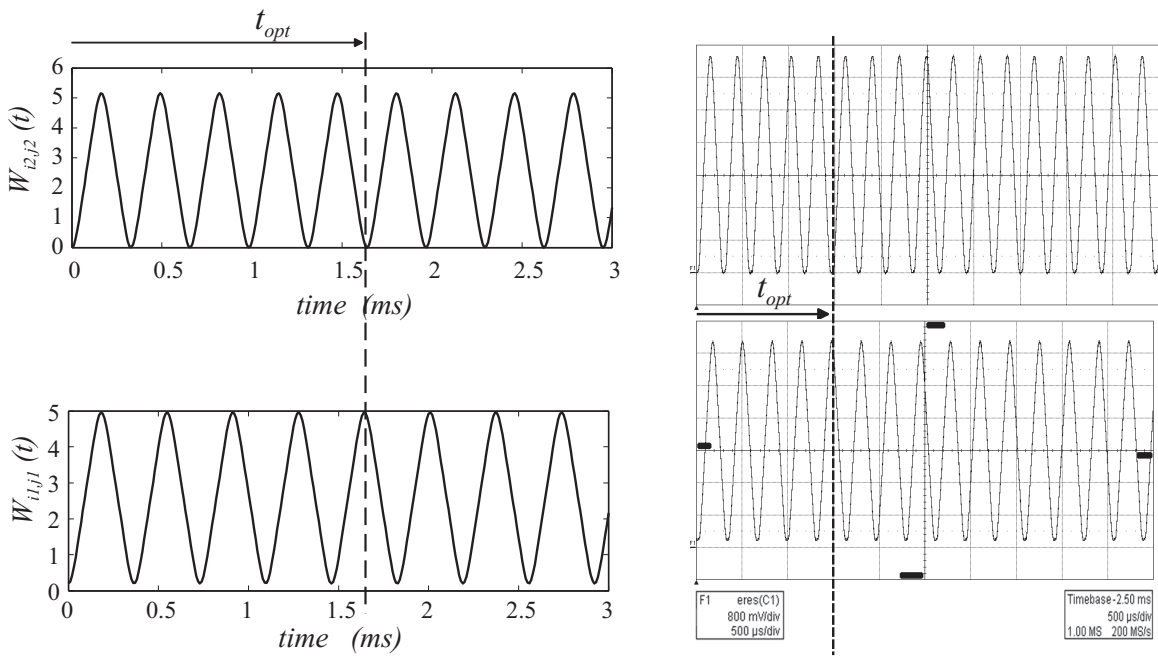

(b)

Fig. 10. Theoretical $(a)$ and experimental $(b)$ voltage temporal evolutions for two oscillators submitted to very narrow initial conditions. Top panel: initial condition is $W_{i 2, j 2}^{0}=0$; Bottom panel: initial condition is $W_{i 1, j 1}^{0}=0.2$. System parameters: $K=10, R=10 K \Omega, C=10 n F$, $m=2.58 \mathrm{~V}, \alpha=1.02 \mathrm{~V}$. 


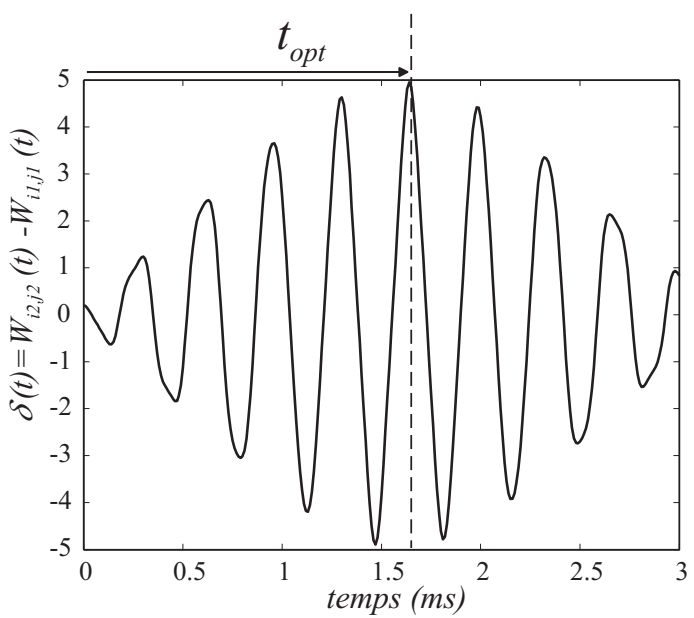

(a)

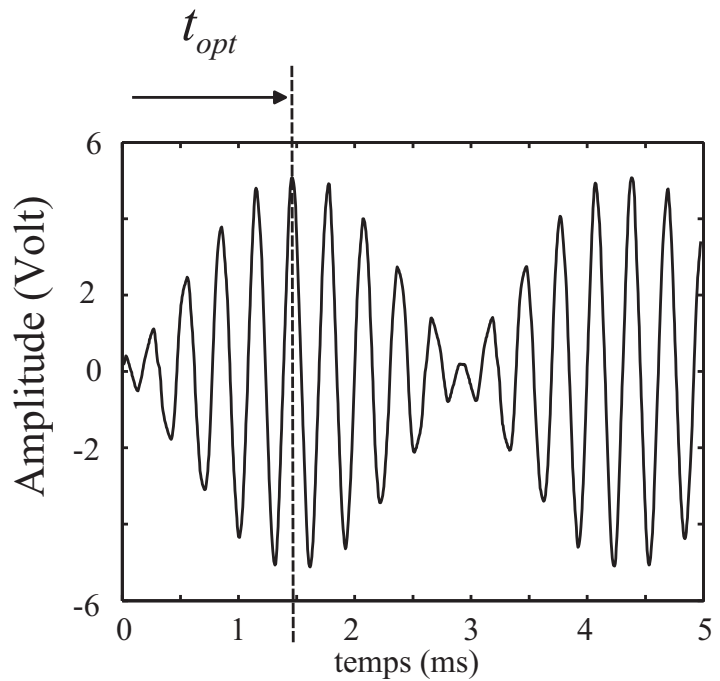

(b)

Fig. 11. Temporal evolution of the amplitude difference between the two oscillators of figure 10. (a) Theoretical evolution; (b) Experimental evolution. System parameters: $K=10$, $R=10 \mathrm{~K} \Omega, C=10 \mathrm{nF}, m=2.58 \mathrm{~V}, \alpha=1.02 \mathrm{~V}$.

Finally, in order to ensure the existence of elliptic Jacobi functions as solutions, modulus $k_{i, j}$ must stay in the $[0 ; 1]$ range. Consequently, the initial conditions applied to the lattice must be smaller than $m-\alpha \sqrt{2}$ or greater than $m+\alpha \sqrt{2}$. These conditions allow an energy greater than the potential barrier energy for all the cells of the network. 


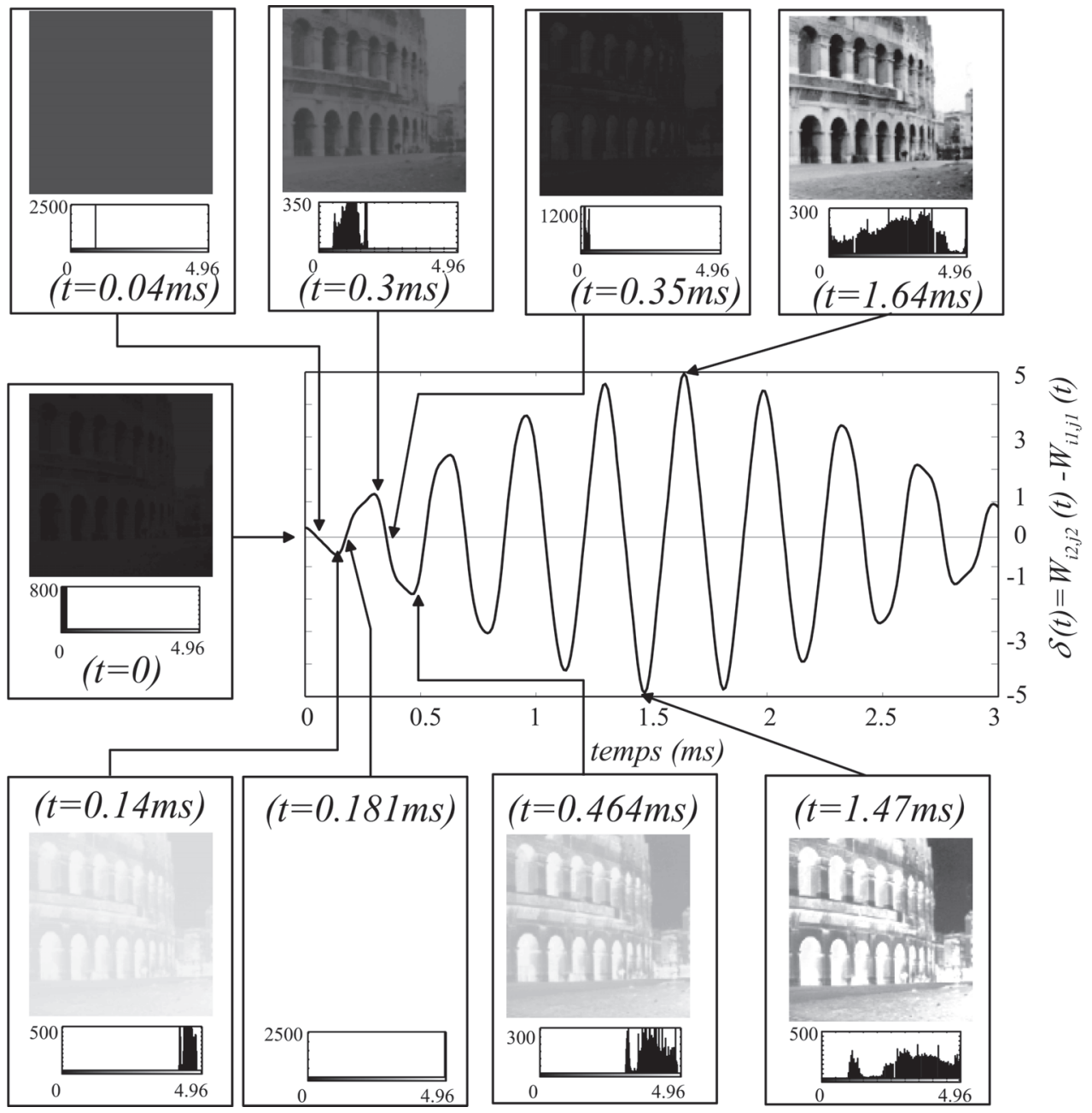

Fig. 12. Time evolution and histogram of a weakly contrasted image. The original image loaded in the network is represented with its histogram at time $(t=0)$ on the left. The evolution of the amplitude difference shows the image dynamics for periodic particular times corresponding to local minima or maxima of contrast, with the possibility to get also image inversion. 
As a result, the weakly contrasted image does not present grey levels exceeding $m-\alpha \sqrt{2}$ for its contrast to be enhanced.

\section{Conclusion}

In this chapter we have presented an overview of nonlinear processing inspired by Reaction-Diffusion and inertial electronic systems. Reaction-Diffusion electrical networks allow noise filtering and image segmentation while Hamiltonian electrical networks enable contrast enhancement. Each network is obtained by coupling elementary cells including classical components such as resistors, capacitors, self inductors, operational amplifiers or analog multipliers.

If the elementary cell can be easily built with such components, implementing the whole network is far from being realistic. We trust that it could be interesting to use Field Programmable Gate Array circuits (FPGA) to enable a large scale integration of the network. However, it requires to re-cast the nonlinear processing algorithms.

Lastly, as revealed by the reaction-diffusion systems, the filtering tasks are strongly depending on the nonlinearity. Therefore, we think that considering different nonlinearities could be the starting point for further development in nonlinear image processing.

\section{References}

Arena, P., Basile, A., Bucolo, M. \& Fortuna, L. (2003). An object oriented segmentation on analog cnn chip, IEEE Trans. on Circuits. and Syst. 50(7): 837-846.

Black, M. J., Sapiro, G., Marimont, D. H. \& Heeger, D. (1998). Robust anisotropic diffusion, IEEE Trans. on Image Processing 3(7): 421-432.

Chua, L. (1999). CNN: A paradigm for complexity in Visions of Nonlinear Science in the 21st Century, World Scientific, Singapore, chapter 13, pp. 793-826.

Chua, L. \& Yang, L. (1988). Cellular neural networks: Theory, IEEE Trans. on Circuits and Syst. 35(10): 1257-1272.

Grassi, G., Di Sciascio, E., Grieco, L. \& Vecchio, P. (2006). New object-oriented segmentation algorithm based on the CNN paradigm, IEEE Trans. on Circ. and Syst.II: Express Brief 53: 259-263.

Grassi, G. \& Grieco, L. A. (2003). Object-oriented image analysis using the cnn universal machine: new analogic cnn algorithms for motion compensation, image synthesis and consistency observation, IEEE Trans. on Circuits. and Syst. 50(4): 488-499.

Julian, P., Dogaru, R. \& Chua, L. (2002). A piecewise-linear simplicial coupling cell for CNN gray-level image processing, IEEE Trans. on Circ. and Syst. 3: 904-913.

Morfu, S. (2005). Image processing with a cellular nonlinear network, Phys. Lett. A 343(4): 281-292.

Morfu, S. (2009). On some applications of diffusion processes for image processing, Phys. Lett. A 373: 2438-2444.

Morfu, S., Marquié, P., Nofiele, B. \& Ginhac, D. (2008). Nonlinear systems for image processing, Advances In Imaging and Electron Physics, Elsevier 152: 79-153.

Morfu, S., Nofiele, B. \& Marquié, P. (2007). On the use of multistability for image processing, Phys. Lett. A 367: 192-198.

Nagy, Z. \& Szolgay, P. (2003). Configurable multilayer cnn-um emulator on fpga, IEEE Trans. on Circ. and Syst.I: Fund. Theory Appl. 50: 774-778. 
Occhipinti, L., Spoto, G., Branciforte, M. \& Doddo, F. (2001). Defects detection and characterization by using cellular neural networks, IEEE Int. Symposium on circuits and systems ISCAS 3: 481-484.

Perona, P. \& Malik, J. (1990). Scale space and edge detection using anisotropic diffusion, IEEE Trans. Pattern Anal. Machine Intell. 12(7): 629-639.

Teuscher, C. \& Adamatzky, A. (2005). Proceedings of the 2005 Workshop on Unconventional computing 2005 from cellular automata to wetware, Luniver Press Beckington, ISBN 978-0-9551170-2-2.

Venetianer, P., Werblin, F., Roska, T. \& Chua, L. (1995). Analogic CNN algorithms for some image compression and restoration tasks, IEEE Trans. on cicuits and syst. 42(5): $278-284$. 


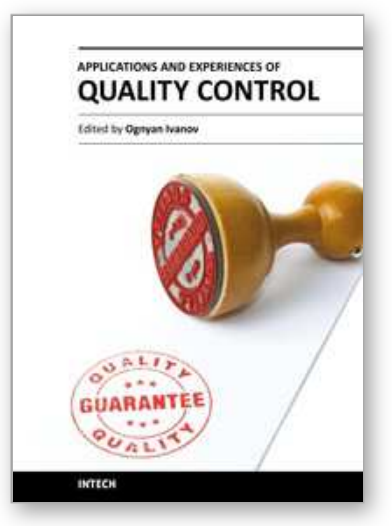

\section{Applications and Experiences of Quality Control \\ Edited by Prof. Ognyan Ivanov}

ISBN 978-953-307-236-4

Hard cover, 704 pages

Publisher InTech

Published online 26, April, 2011

Published in print edition April, 2011

The rich palette of topics set out in this book provides a sufficiently broad overview of the developments in the field of quality control. By providing detailed information on various aspects of quality control, this book can serve as a basis for starting interdisciplinary cooperation, which has increasingly become an integral part of scientific and applied research.

\section{How to reference}

In order to correctly reference this scholarly work, feel free to copy and paste the following:

S. Morfu and P. Marquié (2011). On Some Applications of Nonlinear Differential Equations in Image Processing: Concepts and Electronic Implementation, Applications and Experiences of Quality Control, Prof. Ognyan Ivanov (Ed.), ISBN: 978-953-307-236-4, InTech, Available from:

http://www.intechopen.com/books/applications-and-experiences-of-quality-control/on-some-applications-ofnonlinear-differential-equations-in-image-processing-concepts-and-electronic

\section{INTECH}

open science | open minds

\author{
InTech Europe \\ University Campus STeP Ri \\ Slavka Krautzeka 83/A \\ 51000 Rijeka, Croatia \\ Phone: +385 (51) 770447 \\ Fax: +385 (51) 686166 \\ www.intechopen.com
}

\author{
InTech China \\ Unit 405, Office Block, Hotel Equatorial Shanghai \\ No.65, Yan An Road (West), Shanghai, 200040, China \\ 中国上海市延安西路65号上海国际贵都大饭店办公楼 405 单元 \\ Phone: +86-21-62489820 \\ Fax: $+86-21-62489821$
}


(C) 2011 The Author(s). Licensee IntechOpen. This chapter is distributed under the terms of the Creative Commons Attribution-NonCommercialShareAlike-3.0 License, which permits use, distribution and reproduction for non-commercial purposes, provided the original is properly cited and derivative works building on this content are distributed under the same license. 